\title{
Cloud Computing: Demand and Supply in Banking, Healthcare and Agriculture
}

\author{
Avinash Thakur ${ }^{1}$, Manoranjan Kumar ${ }^{2}$, Rajan Kumar Yadav ${ }^{3}$ \\ ${ }_{1,2,3}$ (Department of Computer, JNTU Hyderabad, India)
}

\begin{abstract}
Right from the Stone Age one law is governing the market for every generation and that well acknowledged law is "Law of Demand \& Supply". ICT is not far away from this law. It is a well-known fact that when cyber world is marching rapidly towards higher form of technological evolution, Cloud Computing has entered as backbone support to ICT as a tool for better cost performance. It is a platform, which is highly governed by the law of "Demand \&Supply", whether it is Banking, Healthcare or Agriculture, all are in need of this technology to make their performance as cost effective as possible and ubiquitous in functionality. As by the law, Demand and Supply both go hand-to-hand. This paper presents some of the demand facets and corresponding supply services for banking, agriculture and healthcare. It enables software architect to make the framework of any sector to build up fully fledged working domain in due consideration of cloud computing.

Keyword: Banking and Financial System (BFS), Cloud Computing, Hospital Management Information System (HMIS), Information Communication Technology (ICT), SaaS Application, Total Cost of Ownership (TCO)
\end{abstract}

\section{Introduction}

The increasing use of IT brought with it overheads in the implementation and maintenance of computing systems. The amount of time and finances invested in managing IT has exponentially increased. It is the demand of the scenario that there must be cost contraction. In the continuous journey of evolution of information communication technology (ICT), various sectors whether it is banking, agriculture [1], healthcare or education, a complete transition is required from on premise to cloud computing environment. Cloud computing has gained huge popularity in industry because of its ability to host applications whose services can be delivered to consumers rapidly at minimal cost [2]. Different application domain can avail advantage from cloud computing not only from scientific application to business but also from consumer applications.

Availability of different types of cloud deployment architectures[2] in the industry creating a tough competition among the service providers in today's competitive market .It is the tough challenge to standardize cloud architecture framework which allows customers the flexibility to move between cloud vendors seamlessly, keeping the cost of the service offering within competitive rates.

In this paper we are presenting few of the glimpses of various sectors in which implementing a cloud will definitely create a high transition, which is not beneficial only for industrial scenario but definitely going to serve humankind also. Among industries sectors, banking, agriculture, healthcare would benefit the most as cloud provide them with the socio economic reach that they lack for the decades. Similarly the cloud promises to deliver affordable, reliable and computing solutions to all these sectors.

\section{Cloud in Banking Sector}

As we embarked into 2014, financial institutions and banking sector are possibly the most advanced in terms of technology adoption and can use cloud as a key differentiator [3][4]. In the current era of banking and financial intermediation the need of high level business process automation, maturity in the functional portfolio, straight through processing and proven technology outsourcing benefits, BFS institutions stand to benefit significantly from cloud computing capabilities [5][6]. To overcome the financial risk and cost issues, bank must devise innovating operating models. An imaginative approach is needed to cut cost, reduce risk and control a disintegrated value chain [7]. 


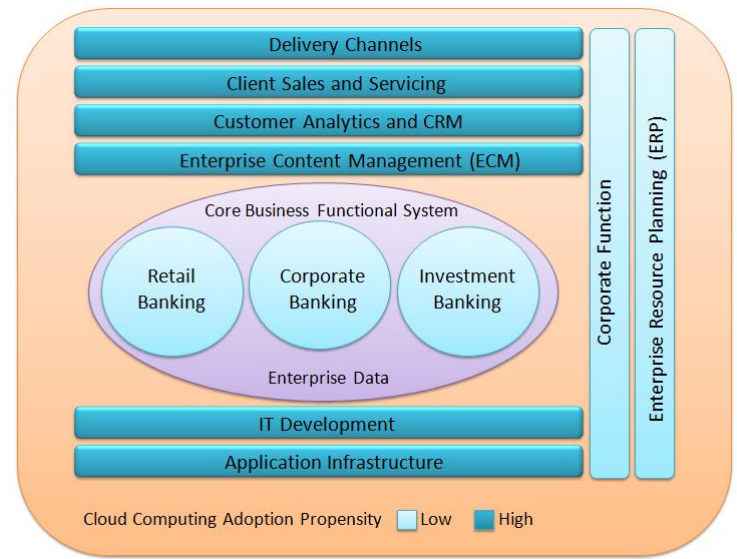

Fig. 2.1 Area Based Suited for Cloud Adoption

As a high quality banking cloud application, anyone has to revisit the existing architecture [3] and enhance the experience of supply side and demand side of the cloud in the sector of banking applications.

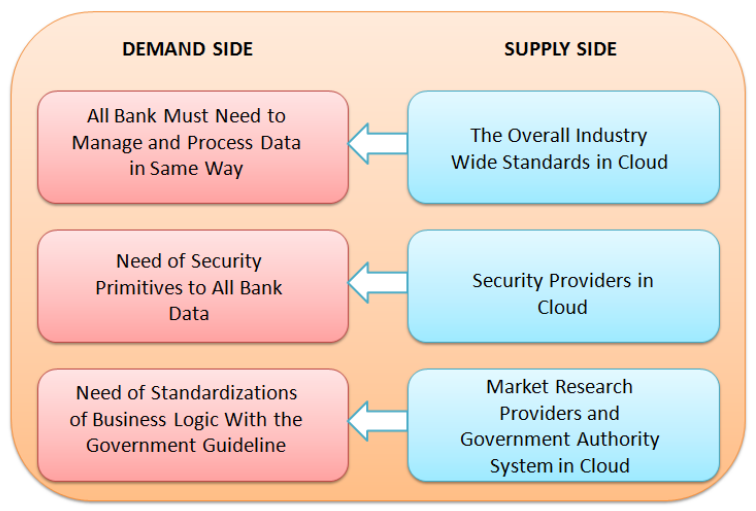

Fig. 2.2 Demand and Supply Scenario in Banking

In above diagram the overall scenario of supply side and demand side shows that how the data can be managed and processed. So, firstly having componentized value chain corresponding IT architectures will require industry-wide data standards. For a scenario customer details and data held by one bank must be held in a format that enables it to be processed simply and accurately by any other bank, financial institutions and central payments facilitator. It will enhance the operational facility for delivery channel to deliver business logic and operational logic.

Secondly, the crucial data and business logic need to be secured in all layer of cloud environment scenario. All data in retail banking, corporate banking and investment banking are very crucial to bank as well as customers. So the supply side must deliver security primitives to all banking and enterprise data in the cloud environment.

Thirdly, at demand side, there is need of information to standardize and analyze the business logic as well as stick to the Government guidelines; on the behalf of demand side cloud environment's supply side must fulfill its need.

So in the supply side there is a requirement of guidelines to be followed which are regulated by the government authority like Reserve Bank of India (RBI) for the entire Bank in the cloud. In this scenario the cloud service providers, Business Logic Company, Market Research Company and government authority give the input to supply side. Therefore by applying all of the above to demand and supply environment of cloud, all the data in retail banking, corporate banking and investment banking can be managed and processed to full-fill all the requirements of banks as well as other financial institutions.

\section{Cloud in Healthcare}

Healthcare is domain where computer technology has found several and diverse applications, from supporting business functions to providing assistance to cure diseases [8].

The healthcare industry has been leveraging technological innovations for decades to provide superior quality services to patients. Medical technology-based devices and equipments such as Computed Tomography 
(CT) Scanners, Diagnostic Sonographic Scanners, Magnetic Resonance Imaging (MRI) Scanner, remote monitoring devices, health and wellness-check devices etc. have helped in diagnosing health problems eliminating the need of expensive and hazardous surgeries.

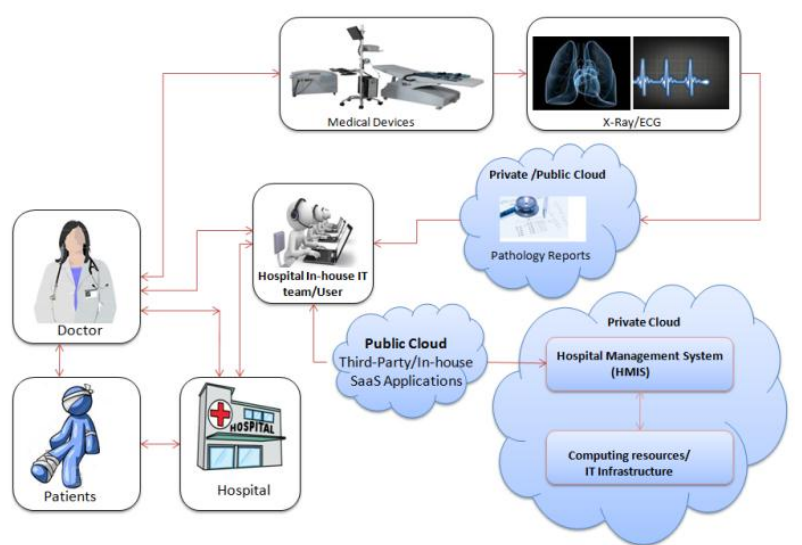

Fig. 3.1 Healthcare Ecosystem Cloud Adoption

Most countries invested significantly in medical technologies and this market is growing rapidly. Information and Communication technology (ICT) has performed a major role in digitizing and communicating patient information, leading to rapid patient diagnosis which further leads to faster time-to treatment and superior overall healthcare [9]. An important application is the use of cloud technology for supporting doctors in providing more effective diagnostic process [10]. The capillary development of internet connectivity and its accessibility from any device at any time has made cloud technologies an attractive option for developing health-monitoring system [11] [12].

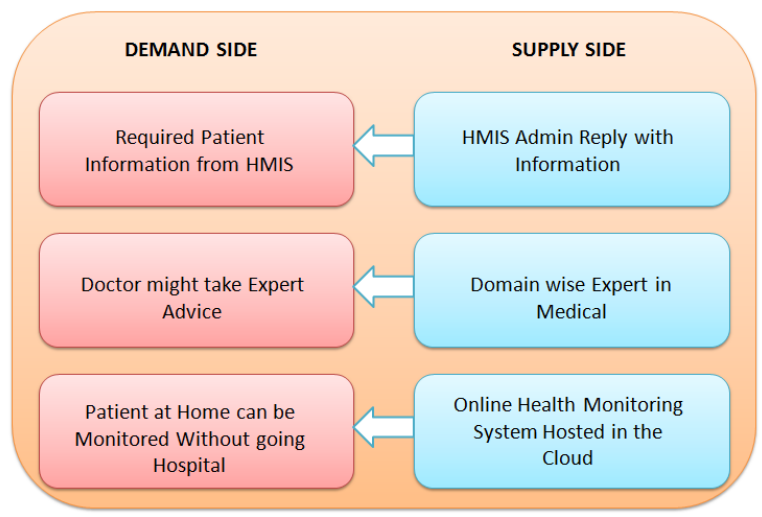

Fig. 3.2 Demand and Supply Scenario in Healthcare

In healthcare provisioning process, once the patient is admitted into the hospital and his details are entered in the Hospital Management Information System (HMIS) the process of diagnosis begins. Now let us consider scenario when doctors are standing in demand side, he wants to access information about patient from Hospital Management Information System. Doctor will consult to admin of HMIS and admin will reply with complete information about patient. In the process, the doctor might take expert advice as and when required. And cloud expert will reply with expert advice. Cloud computing technologies allow the remote monitoring of a patient's Heartbeat data, its analysis in minimum time, and the notification of first-aid personnel. This way a patient at risk can be constantly monitored without going to hospital.

The cloud has already started delivering value to the healthcare industry. Radiology departments are amongst some of the early adopters. They are exploiting the massive computing capability made available through the cloud. Hospitals and laboratories are being charged per image for their storage requirements. The stored images are not restricted within geographic boundaries. They are accessible from anywhere and anytime to the clinicians or physicians to make informed decisions.

The cloud can also help reduce the overall Total Cost of Ownership (TCO) for healthcare service providers, which could go down between 10 to 30 percent according to certain estimates. 


\section{Cloud in Agriculture}

In spite of the various ICT projects in India, the country is still facing various challenges:

- $\quad$ Lack of awareness among farmers about the benefits of ICT in agriculture.

- Deficient production information.

- Inadequate knowledge about the weather forecast, threats from pests and diseases.

Cloud computing is going to solve these challenges .By using the cloud technology, farmers have nothing to worry about hardware and software investments and also the technical knowledge to learn them [14][15]. Farmer as client need to send a request to the service provider and by thorough analysis of request, result will be passed back to the client. Cloud computing will give on demand opportunities through which data sharing and data collection is possible, which is very crucial for agricultural research and development. This technology can offer a centralized knowledge database which can be used to store all the agriculture related information.

Some of the possible solutions which can be provided by service providers to the farmers are:

- $\quad$ Prediction of weather and related knowledge database.

- Database for crop related information.

- Database for market related information.

- Database for production related data.

Farmers can also post their problems seeking for solutions from the expert .With the help of mobile phone applications farmers can instantly contribute to the knowledge database by uploading crop and soil related information, pictures, videos and any other information.

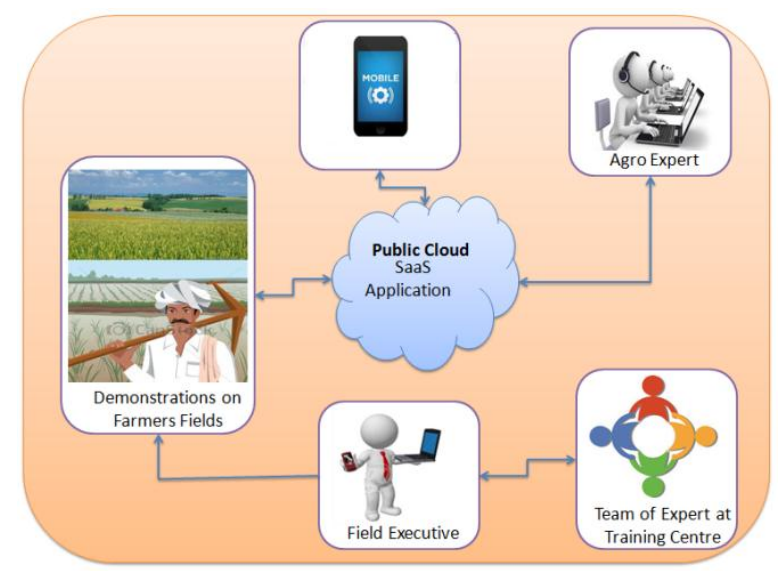

Fig. 4.1 Agriculture Ecosystem Cloud Adoption

Let us consider the scenario when farmers are standing in demand side; they require information about quality of the soil and use of fertilizer according to the quality of the soil. Farmers will consult to field executive and the executive will send the required information to the team of experts and agro experts.

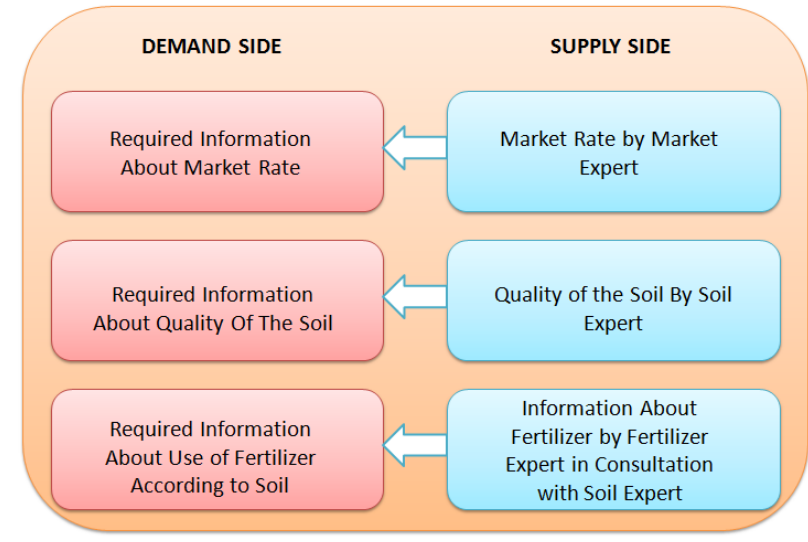

Fig. 4.2 Demand and Supply Scenario in Agriculture 
By analyzing all the details related to the fields, solutions will be given to the farmers via field executive. By using this technology we are not only supporting our farmers in building their work technically but abolition of middle men is also possible for market related work.

By having brief overview of the environment, this architectural implementation is definitely going to take giant leap in the agricultural field of the country and will create a difference in the agro market .It will ease the way of work and what is happening today. By implementing this techno compatible platform, it is going to be a boon for the agriculture.

\section{Conclusion}

In this paper authors try to give an overview of some of the issues and challenges faced by banking, healthcare and agriculture sector. Later on with efficient coordination to issues and challenges, efforts are taken to balance demand and supply scenarios of above mentioned sectors. We tried to propose some of the demand sides of the various sectors and provided the solution via supply side in cloud computing environment. Any time service, from anywhere, is possible by adopting this technology. It also serves the purpose of cost effectiveness when the IT world gets trapped in recession. Giving economical effectiveness to any platform must be a high concern for all the contributors. Cloud Computing plays a vital role for supporting the humankind in the field of agriculture, banking and health care services.

\section{Acknowledgements}

We wish to express our sincere gratitude to Mihir Kumar Jha. This research paper would not have been possible without the commitment and contributions from him. He is a professional banker currently working with Central Bank of India. He looks after Market Risk in the Risk Management vertical of the Bank. He has done his Post Graduation in Banking and Finance from National Institute of Bank Management, Pune, a premiere Institute governed by Reserve Bank of India. His area of interest is Credit and Market Risk management for Financial Institution.

\section{Reference}

[1] Yanxin Zhu, Di Wu and Sujian Li, Cloud Computing and Agricultural Development of China: Theory and Practice, IJCSI International Journal of Computer Science Issues, Vol. 10, Issue 1, No 1, January 2013

[2] Rajan Kumar Yadav, Avinash Thakur, Manoranjan Kumar, Cloud Computing: Environment of Next Generation Technology, IOSR Journal of Computer Engineering (IOSR-JCE), e-ISSN: 2278-0661, p-ISSN: 2278-8727Volume 16, Issue 2, Ver. IX (Mar-Apr. 2014), PP144-148

[3] Sudhir Sriram, Cloud Computing in Banking, Capgemini Financial services, Copyright @ 2011 Capgemini

[4] www.capgemini.com/financialservices

[5] Abhinav Garg,CLOUD COMPUTING FOR THE FINANCIAL SERVICES INDUSTRY, (C) 2011 Sapient Corporation

[6] www.sapientglobalmarkets.com

[7] Banking takes to Cloud Cloud based security services, (C) 2012 Netmagic Solutions Pvt. Ltd

[8] Mark Grindle, Jitendra Kavathekar, Dadong Wan, A new era for the healthcare industry Cloud computing changes the game, Accenture 2013

[9] How Cloud Computing can Help Solve Healthcare's Looming IT Crisis, Solution Brief, Intel Hardware-assisted Security, Intel Xeon Processor E5 family, Healthcare Cloud Computing

Impact of Cloud Computing on Healthcare, Cloud Standards Customer Council, 2012

[11] Dion Alley, Tariq Ahmed, Jack Androvich, Scott Archibald, The Cloud Computing Guide for Healthcare, Focus Research,2012

[12] Your Cloud in Healthcare, Industry brief White Paper,VM Ware, 2011

[13] http://www.vmware.com/solutions/industry/healthcare/

[14] Karuna ChandraulA், and Archana Singh, An Agriculture Application Research on Cloud Computing, International Journal of Current Engineering and Technology, ISSN 2277 - 4106 @ 2013 INPRESSCO

[15] Dr. G Sahoo, Dr. Shabana Mehfuz and Rashmi Rai, Applications of Cloud Computing for Agriculture Sector, CSI Communications , November 2013

[16] Miao Tian, Qingli Xia and Hao Yuan Zhongnan, Discussion on the Application of Cloud Computing in Agricultural Information Management, Research Journal of Applied Sciences, Engineering and Technology 5(8): 2538-2544, 2013 ISSN: 2040-7459; eISSN: 2040-7467 (C) Maxwell Scientific Organization, 2013

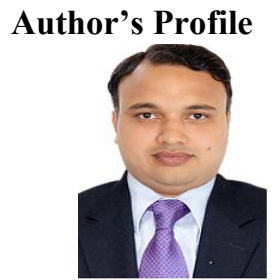

Avinash Thakur. He has received B.E. degree from MIT Pune, India. He is pursuing M. Tech in Computer Science and Engineering from Jawaharlal Nehru Technological University (JNTU), Hyderabad, India. His area of interest is Computer Network, Digital Signal Processing, Mobile Computing, Theory of Computation, Cloud Computing and Engineering Mechanics. He is having 5-years of teaching experience in the field of Engineering Mechanics. 
Manoranjan Kumar. He has received B.E. degree from MIT Pune, India. He is pursuing M. Tech in Computer Science and Engineering from Jawaharlal Nehru Technological University (JNTU), Hyderabad, India. His area of interest is Data Structure, Compiler Design, Theory of Computation, Digital Signal Processing and Programming Languages.

Rajan Kumar Yadav. He is an IBM DB2 Academic Associate. He has received B.E. degree from MIT Pune, India. He is pursuing M. Tech in Computer Science and Engineering from Jawaharlal Nehru Technological University (JNTU), Hyderabad, India. His area of Interest is Cloud Computing, Programming Languages and Database. He has done various research works in his area of research to contribute to IT industry and in the field of Open Source Technology. 